\title{
Cross-sectional Study of Patients with Diffuse Large B-Cell Lymphoma: Assessing the Effect of Host Status, Tumor Burden, and Inflammatory Activity on Venous Thromboembolism
}

\author{
Sung Hee Lim, MD ${ }^{1}$ \\ Sook-young Woo, BS² \\ Seonwoo Kim, PhD2 \\ Young Hyeh Ko, MD, PhD ${ }^{3}$ \\ Won Seog Kim, MD, PhD 1 \\ Seok Jin Kim, MD, PhD1
}

\author{
${ }^{1}$ Division of Hematology-Oncology, \\ Department of Medicine, \\ Samsung Medical Center, \\ Sungkyunkwan University \\ School of Medicine, Seoul, ${ }^{2}$ Biostatistics Team, \\ Samsung Biomedical Research Institute, \\ Seoul, ${ }^{3}$ Department of Pathology, \\ Samsung Medical Center, \\ Sungkyunkwan University \\ School of Medicine, Seoul, Korea
}

\author{
Correspondence: Seok Jin Kim, MD, PhD \\ Division of Hematology-Oncology, \\ Department of Medicine, \\ Samsung Medical Center \\ Sungkyunkwan University School of Medicine, \\ 81 Irwon-ro, Gangnam-gu, Seoul 06351, Korea \\ Tel: 82-2-3410-1766 \\ Fax: 82-2-3410-1754, \\ E-mail: kstwoh@skku.edu \\ Received September 22, 2014 \\ Accepted December 9, 2014 \\ Published Online March 2, 2015
}

\begin{abstract}
Purpose
The risk factors for venous thromboembolism (VTE) in diffuse large B-cell lymphoma (DLBCL) are not clear although thrombosis can be associated with host status, tumor burden, and inflammatory activity. We assessed the effect of those factors on VTE in a cross-sectional study of patients enrolled in a prospective cohort study.
\end{abstract}

\section{Materials and Methods}

We analyzed the occurrence of VTE in 322 patients with newly diagnosed DLBCL who received rituximab with cyclophosphamide, doxorubicin, vincristine, and prednisone (R-CHOP) between 2008 and 2011. Serum levels of inflammatory cytokines were measured from serum samples archived at diagnosis.

\section{Results}

With a median follow-up duration of 41.9 months, VTE was documented in 34 patients (10.6\%). A comparison of baseline characteristics indicated the group with VTE had higher percentage of old age, stage III/IV and extranodal involvements than the group without VTE $(p<0.05)$. Thus, the International Prognostic Index was significantly associated with VTE, but the Khorana score was not. A univariate competing risk factor analysis for VTE revealed that increased levels of inflammatory cytokines such as interleukin (IL)- 6 and IL-10 were also associated with VTE $(p<0.05)$ in addition to host and tumor burden. However, a multivariate analysis showed that two host factors including age ( $\geq 60$ years) and poor performance were independent risk factors for VTE.

\section{Conclusion}

Among potential risk factors for VTE including tumor burden and inflammatory activity, age and performance status had a strong impact on the occurrence of VTE in patients with DLBCL who received R-CHOP.

\section{Introduction}

Venous thromboembolism (VTE), including deep vein thrombosis (DVT) and pulmonary embolism (PE), is a wellknown adverse event in patients with cancer. Their risk of VTE is higher than in the general population because cancer and treatments such as chemotherapy raise the risk of VTE. However, the incidence of VTE is varies by the type of cancer, because each type of tumor cell might have different

\section{Key words}

Diffuse large B-cell lymphoma, Venous thromboembolism, Risk factors, Chemotherapy procoagulant activity and their treatment poses differing risks for VTE. Lymphoma is as a high-risk cancer for VTE as patients with lymphoma show a high incidence [1,2]. However, the risk of VTE in such patients can be different by the subtype of lymphoma, because this cancer has many subtypes requiring different treatment approaches. Thus, the exact assessment of the incidence of VTE and analysis for risk factors focusing on the most common subtype of lymphoma might be helpful for managing VTE in patients with lymphoma, and provide reference values for the other 
subtypes of such patients.

Diffuse large B-cell lymphoma (DLBCL) is the most common subtype of non-Hodgkin lymphoma (NHL), accounting for $30 \%-40 \%$ of cases of NHL in Western and Asian countries [3,4]. Since rituximab-an anti-CD20 monoclonal antibody targeting B cells-was introduced for the treatment of B-cell lymphomas, the combination of rituximab with cyclophosphamide, doxorubicin, vincristine, and prednisone (R-CHOP) has become the standard therapy for patients with DLBCL $[5,6]$. Therefore, we drew a crosssectional sample of patients with newly diagnosed DLBCL who were uniformly treated with R-CHOP and registered into our prospective cohort study. We analyzed the influence of host- and disease-related factors on the occurrence of VTE to confirm reports suggesting associations with host factors such as poor performance status and old age, and with disease-related factors such as a high tumor burden $[7,8]$. In addition, we evaluated the association of inflammation with the occurrence of VTE by analyzing laboratory parameters reflecting inflammatory activity such as leukocyte and platelet counts, and C-reactive protein (CRP) and albumin levels. This was because the contribution of tumor burden to the development of VTE might be associated with a local inflammatory microenvironment. Indeed, inflammation promotes thrombosis, and a recent study using two inflammation-associated indexes-the neutrophil/lymphocyte and platelet/lymphocyte ratios-showed strong associations with the risk of VTE in ambulatory patients with solid cancers [9]. This association between inflammation and VTE might be related with an elevated level of inflammatory cytokines such as interleukin (IL)-6 and tumor necrosis factor $(\mathrm{TNF}) \alpha[10]$. In addition, there were more candidate inflammatory mediators for venous thrombotic disease [11-13]. Considering that VTE commonly occurs at diagnosis or during an early period of treatment $[1,2,7,14,15]$, we also measured pretreatment levels of inflammatory cytokines to explore their impact on the occurrence of VTE in patients with DLBCL treated with R-CHOP.

\section{Materials and Methods}

\section{Patients and study design}

This study was a cross-sectional analysis of the occurrence of VTE in patients who were newly diagnosed with DLBCL at the Samsung Medical Center between October 2008 and December 2011. Among 953 patients registered in a prospective cohort during the study period, we selected 322 patients who had available serum samples archived at diagnosis. For this analysis, all patient data were updated in February 2014. The pathology of DLBCL was confirmed by an expert hematopathologist (Y.H.K.) using the World Health Organization classification criteria. All patients were enrolled in our prospective cohort study (NCT No. 00822731), and their clinical information and laboratory results were registered at diagnosis. Prior to treatment and after we had obtained written informed consent, serum samples were archived at $-80^{\circ} \mathrm{C}$. All patients received R-CHOP chemotherapy every three weeks as follows: rituximab $375 \mathrm{mg} / \mathrm{m}^{2}$; cyclophosphamide $750 \mathrm{mg} / \mathrm{m}^{2}$; doxorubicin $50 \mathrm{mg} / \mathrm{m}^{2}$; vincristine 1.4 $\mathrm{mg} / \mathrm{m}^{2}$ (maximum, $2 \mathrm{mg}$ ) intravenously on day 1 ; and prednisone $100 \mathrm{mg} /$ day orally on days $1-5$. We assessed both symptomatic as well as asymptomatic incidental VTE. The occurrence of VTE included PE and/or DVT diagnosed via computed tomography and duplex ultrasonography. We explored the impact of host factors such as age, gender, performance status, and body mass index (BMI) as well as of disease-related factors such as stage, number of extranodal sites, and serum lactate dehydrogenase levels on VTE (Fig. 1). We also analyzed the association of the Khorana score risk prediction model for chemotherapy associated with VTE because all patients received systemic chemotherapy. The Khorana score was calculated according to pretreatment hematology values and BMI as reported $[16,17]$. The impact of the International Prognostic Index (IPI) reflecting tumor burden and host status on VTE was also evaluated and compared with that of the Khorana score. To explore the effect of cytokines on the occurrence of VTE, we also measured the levels of pro/anti-inflammatory cytokines in archived frozen serum samples. The Institutional Review Board of Samsung Medical Center approved this study.

\section{Multiplex cytokine assays}

Serum aliquots were not thawed before we performed the multiplex cytokine assay. We measured the levels of regulated on activation, normal $\mathrm{T}$ cell-expressed and secreted (RANTES), IL-10, IL-6, interferon gamma-induced protein 10 (IP-10), and TNF- $\alpha$ with the Procarta cytokine profiling kit (Panomics, Fremont, CA) according to the manufacturer's instructions, in duplicate. We also measured the level of plasminogen activator inhibitor 1 (PAI), a procoagulant parameter, with the same kit. Based on the cutoff value of each cytokine examined, patients were categorized into high and low groups. The optimal cutoff value for TNF- $\alpha$ was determined by receiver-operating characteristic (ROC) curve analysis. The area under the ROC curve was 0.66 (cutoff value, 0.285 ; $95 \%$ confidence interval [CI], 0.517 to 0.694 ). Because of the low sensitivity and specificity for other cytokines and PAI through ROC curve analysis, we used the median value as the cutoff point for PAI, RANTES, IL-10, 


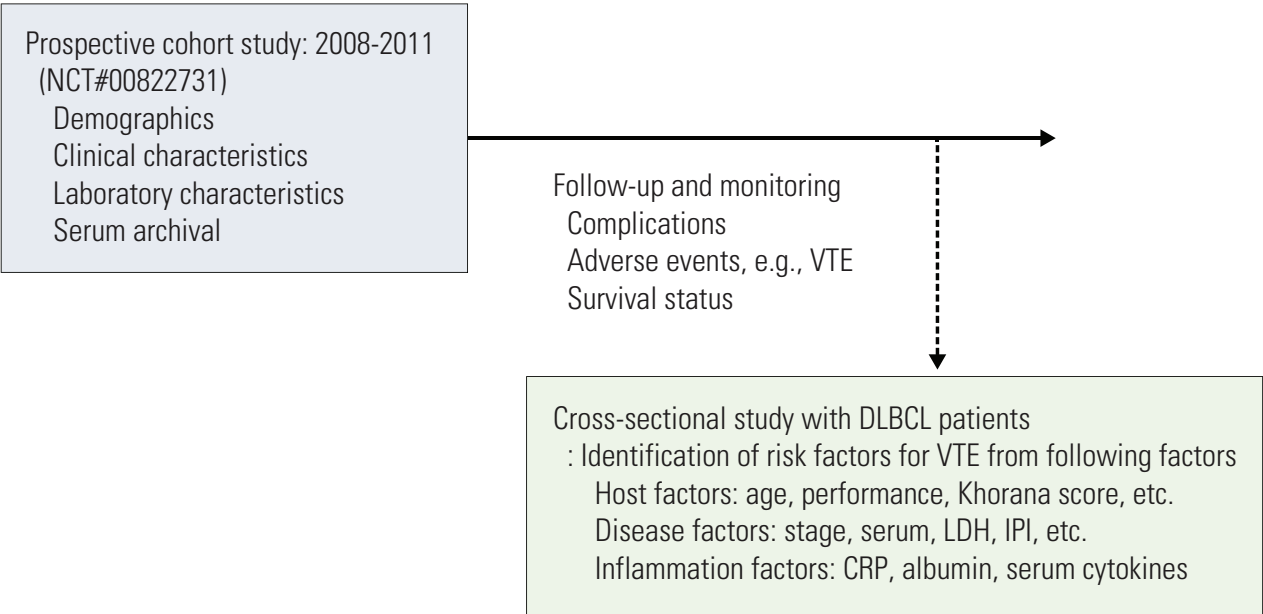

Fig. 1. Study design: cross-sectional analysis. This prospective cohort study aimed to develop a predictive model for treatment outcomes and treatment-related morbidity (such as VTE in this study) in patients with lymphoma. VTE, venous thromboembolism; DLBCL, diffuse large B-cell lymphoma; LDH, lactate dehydrogenase; IPI, International Prognostic Index; CRP, C-reactive protein.

and IP-10 analyses. Only $26 \%$ of all patients showed a positive value of cytokine IL-6, so we categorized the IL-6 level as detectable or undetectable.

\section{Statistical analysis}

Baseline characteristics were compared between the patients with and without VTE by chi-squared and Fisher exact test (when we expected fewer than five counts in any one cell of the contingency table). A ROC curve analysis for the serum cytokine assay was performed using the MedCalc ver. 12.7 (MedCalc Software, Ostend, Belgium). Gray's test was used to compare the cumulative incidence curves for VTE in the presence of the competing risk of death. We used the Fine and Gray regression model to assess a risk factor of interest after adjusting for other potential risk factors in the model. Multivariate analysis was done with selected variables that were significant in univariate analysis. The median follow-up duration was calculated by the Kaplan-Meier estimate of potential follow-up, as reported [18]. Overall survival time was calculated from the date of diagnosis to the date of final follow-up or death from any cause. The Kaplan-Meier estimate was used to compare survival between patients with or without VTE, and the log-rank test was also used to estimate statistical significance between survival curves. Two-sided null hypotheses of no difference were rejected if p-values were less than 0.05 (SAS ver. 9.1.3, Stata ver. 11; SAS Institute, Cary, NC).

\section{Results}

\section{Patient characteristics}

The median follow-up of 322 patients was 41.9 months (95\% CI, 40.6 to 43.2 months), and their median age at diagnosis was 56 years (range, 16 to 86 years). Most patients had good performance status (Eastern Cooperative Oncology Group [ECOG] grade 0/1, 84.5\%). Half of the patients $(46.6 \%)$ presented with Ann Arbor stage III/IV, and more than $30 \%$ of patients had more than two extranodal involvements (Table 1). As a result, 108 patients had high or highintermediate risk on the IPI. Patients were dichotomized into obese and non-obese groups based on a BMI cutoff value of $25 \mathrm{~kg} / \mathrm{m}^{2}$, because few patients had a BMI greater than 35 $\mathrm{kg} / \mathrm{m}^{2}$, the cutoff for the Khorana score. In addition, the number of patients whose pretreatment hematology results fulfilled the criteria of the Khorana score was extremely low: only 16 patients had a high-risk category $(\geq 3)$ in this score. All patients had a central venous catheter installed before starting the first cycle of R-CHOP chemotherapy (median 6 cycles; range, 3 to 8 cycles). Only a few patients received additional therapy such as radiotherapy or surgery (Table 1 ). Most patients were treated as outpatients, and none received prophylactic anticoagulants for VTE during their treatment.

\section{Venous thromboembolism}

VTE was documented in 34 of the 322 patients $(10.6 \%)$, and 
Table 1. Patient characteristics

\begin{tabular}{|c|c|c|c|c|c|}
\hline Characteristic & & No. & VTE $(-)$ & VTE (+) & p-value \\
\hline \multicolumn{6}{|l|}{ Host factor } \\
\hline \multirow[t]{2}{*}{ Age (yr) } & $\leq 60$ & 200 & $185(92)$ & $15(8)$ & 0.022 \\
\hline & $>60$ & 122 & $103(84)$ & $19(16)$ & \\
\hline \multirow[t]{2}{*}{ Gender } & Male & 187 & $166(89)$ & $21(11)$ & 0.645 \\
\hline & Female & 135 & $122(90)$ & $13(10)$ & \\
\hline \multirow[t]{2}{*}{ ECOG PS } & $0 / 1$ & 272 & $247(91)$ & $25(9)$ & 0.062 \\
\hline & $\geq 2$ & 50 & $41(82)$ & $9(18)$ & \\
\hline \multirow[t]{2}{*}{ Smoking } & Never & 221 & $199(90)$ & $22(10)$ & 0.602 \\
\hline & Ever & 108 & $96(89)$ & $12(11)$ & \\
\hline \multirow[t]{2}{*}{ Alcohol } & Non-drinker & 184 & $164(89)$ & $20(11)$ & 0.834 \\
\hline & Drinker & 138 & $124(90)$ & $14(10)$ & \\
\hline \multirow[t]{2}{*}{ Body mass index $\left(\mathrm{kg} / \mathrm{m}^{2}\right)$} & $<25$ & 210 & $188(89)$ & $22(11)$ & 0.947 \\
\hline & $\geq 25$ & 112 & $100(89)$ & $12(11)$ & \\
\hline \multicolumn{6}{|l|}{ Disease-related factor } \\
\hline \multirow[t]{2}{*}{ Serum LDH } & Normal & 161 & $148(91)$ & $13(8)$ & 0.147 \\
\hline & Increased & 161 & $140(87)$ & $21(13)$ & \\
\hline \multirow[t]{2}{*}{ Ann Arbor stage } & I/II & 172 & $163(95)$ & $9(5)$ & 0.001 \\
\hline & III/IV & 150 & $125(83)$ & $25(17)$ & \\
\hline \multirow[t]{2}{*}{ Bone marrow invasion } & Absence & 293 & $263(90)$ & $30(10)$ & 0.552 \\
\hline & Presence & 29 & $25(86)$ & $4(14)$ & \\
\hline \multirow[t]{2}{*}{ B Symptoms } & Absence & 243 & $219(90)$ & $24(10)$ & 0.485 \\
\hline & Presence & 79 & $69(87)$ & $10(13)$ & \\
\hline \multirow[t]{2}{*}{ Extranodal involvement } & $0 / 1$ & 219 & $204(93)$ & $15(7)$ & 0.002 \\
\hline & $\geq 2$ & 103 & $84(82)$ & $19(18)$ & \\
\hline \multicolumn{6}{|l|}{ Pretreatment hematology } \\
\hline \multirow[t]{2}{*}{ Hemoglobin $(\mathrm{g} / \mathrm{dL})$} & $\geq 10$ & 276 & $249(90)$ & $27(10)$ & 0.267 \\
\hline & $<10$ & 46 & $39(85)$ & $7(15)$ & \\
\hline \multirow[t]{2}{*}{ Platelet $\left(\times 10^{9} / \mathrm{L}\right)$} & $<350,000$ & 286 & $257(90)$ & $29(10)$ & 0.480 \\
\hline & $\geq 350,000$ & 36 & $31(86)$ & $5(14)$ & \\
\hline \multirow[t]{2}{*}{ White blood cell $\left(/ \mathrm{mm}^{3}\right)$} & $\leq 11,000$ & 297 & $269(91)$ & $28(9)$ & 0.023 \\
\hline & $>11,000$ & 25 & $19(76)$ & $6(24)$ & \\
\hline \multirow[t]{2}{*}{ C-Reactive protein (mg/dL) } & Normal & 270 & $154(57)$ & $116(43)$ & 0.274 \\
\hline & Increased & 32 & $15(47)$ & $17(53)$ & \\
\hline \multirow[t]{2}{*}{ Albumin (g/dL) } & Normal & 253 & $228(90)$ & $25(10)$ & 0.449 \\
\hline & Decreased & 69 & $60(87)$ & $9(13)$ & \\
\hline \multicolumn{6}{|l|}{ Treatment-related factor } \\
\hline \multirow[t]{2}{*}{ Radiotherapy } & Done & 43 & $38(88)$ & $5(12)$ & 0.806 \\
\hline & Not done & 279 & $250(90)$ & $29(10)$ & \\
\hline \multirow[t]{2}{*}{ Surgery } & Done & 43 & $39(91)$ & $4(9)$ & 0.773 \\
\hline & Not done & 279 & 249 (89) & $30(11)$ & \\
\hline
\end{tabular}

VTE, venous thromboembolism; ECOG PS, Eastern Cooperative Oncology Group performance status; LDH, lactate dehydrogenase.

the 1 year actuarial incidence rate was 9.8\%. Most cases of VTE occurred within 3 months of diagnosis, including five cases of VTE observed at the time of DLBCL diagnosis. Thus, the median time to the occurrence of VTE was 2.35 months (range, 0 to 29.4 months). After the completion of six cycles of R-CHOP chemotherapy, only four cases of VTE were observed: one case of symptomatic DVT in lower legs (1 month after the 6th R-CHOP), two cases of PE (6 months after the 6th cycle: one with PE plus DVT and the other with $\mathrm{PE}$ only), and one case of splenic vein thrombosis that was 


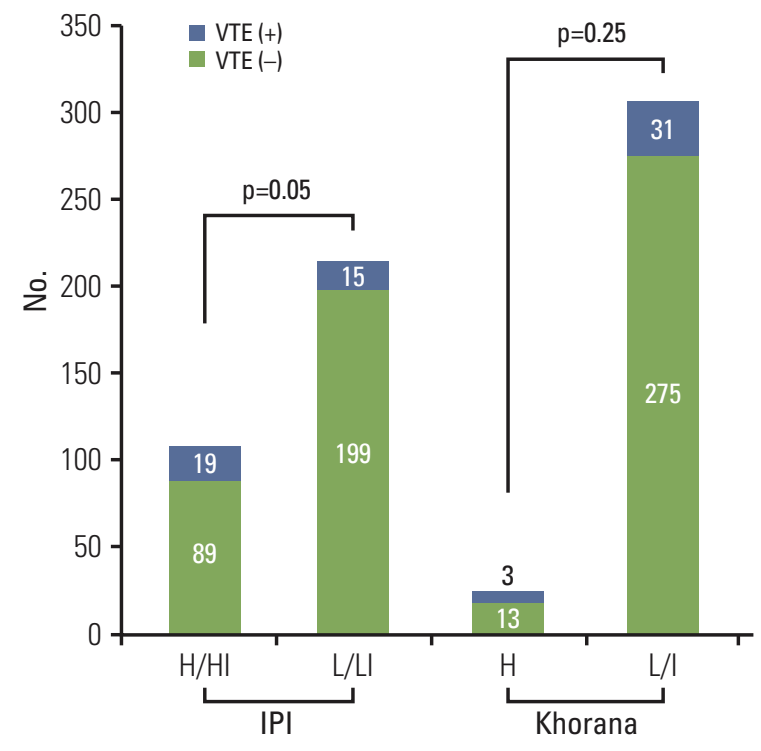

Fig. 2. Comparison of International Prognostic Index (IPI) and Khorana score on the occurrence of venous thromboembolism (VTE). Patients with high or high-intermediate $(\mathrm{H} / \mathrm{HI})$ IPI risk $(18 \%, 19 / 108)$ had significantly more VTE events than those with low or low-intermediate (L/LI) IPI risk $(7 \%, 15 / 214 ; \mathrm{p}=0.05)$. Khorana risk classification did not show significant association with VTE (Khorana score high [ $\geq 3]$ group [H], 24\%; low/intermediate group [L/I], 10\%; $\mathrm{p}=0.25)$.

detected by follow-up computed tomography (CT) scan (1 year after the 6th cycle). Eighteen of these 34 patients (52.9\%) had a DVT, 12 patients $(35.3 \%)$ had a PE and three patients $(8.8 \%)$ had a PE with a DVT. Although all patients received a central venous catheter, only three patients had an upper limb DVT associated with it. Among those patients who developed VTE, two-thirds $(23 / 34,67.6 \%)$ had VTEassociated symptoms; the other cases were diagnosed incidentally by radiology imaging during staging work-up or response evaluation. Sixteen patients $(16 / 34,47 \%)$ were treated with anti-coagulant therapy using low molecular weight heparin for two patients, intravenous heparin for one patient and warfarin for 13 patients. Eleven patients who were asymptomatic but incidentally diagnosed with minimal PE and/or DVT had no anti-coagulant treatment. Four patients who had vague symptoms associated with VTE were not treated with anticoagulant, and three patients with venous compression caused by lymphoma mass were initially treated with chemotherapy only without anticoagulant therapy.

\section{Comparison of patients with and without VTE}

Comparisons of host factors revealed that the proportion of patients older than 60 years $(56 \%, 19 / 34)$ was significantly higher in patients with VTE than in patients without it $(36 \%$, $103 / 288, p=0.022$ ) (Table 1). Among disease-related factors, tumor burden (stage III/IV, extranodal involvement) was significantly higher in patients with VTE $(73 \%$ and $56 \%$, respectively) than in patients without VTE ( $43 \%$ and $29 \%$, respectively). As a result, the number of patients with VTE was significantly higher in the IPI high or high-intermediate risk groups $(18 \%, 19 / 108)$ than in the low or low/intermediate risk groups $(7 \%, 15 / 214)$ (Fig. 2). Of the parameters in the Khorana score, only the white blood cell (WBC) count was significantly associated with VTE. Accordingly, the Khorana risk classification was not significantly associated with VTE in this study (Fig. 2). Among inflammatory markers, serum albumin and CRP levels did not differ significantly between patients with and without VTE. A direct comparison of the mean levels of cytokines including RANTES, IL-10, IP-10, IL- 6 , and TNF- $\alpha$ as well as procoagulant PAI- 1 did not differ significantly (Fig. 3).

\section{Competing risk factor analysis for VTE}

We performed competing risk factor analysis with variables including host- and disease-related factors and inflammatory markers (Table 2). Among host factors, age older than 60 years was significantly associated with a risk of VTE $(\mathrm{p}=0.03)$ whereas performance status $(\mathrm{ECOG} \geq 2)$ and BMI $\geq 25$ only showed a tendency for developing VTE ( $p=0.06$ ). Stage III/IV and more than two extranodal involvements were significantly associated with VTE. As a result, patients who belonged to the IPI high and high-intermediate risk groups more frequently developed VTE than did low and low/intermediate risk groups $(\mathrm{p}<0.05)$ (Table 2). Among inflammation-related laboratory parameters, only a high WBC count $\left(>11 \times 10^{9} / \mathrm{L}\right)$ was associated with VTE. When patients were dichotomized into low and high groups of serum cytokines, high levels of RANTES, IL-10, and IP-10 were significantly associated with VTE. Patients with detectable IL-6 were also more likely to develop VTE (Table 2). Cumulative incidence curves for thrombosis differed significantly between age, ECOG performance status, stage, number of extranodal involvement, IL-6 and IP-10 (Fig. 4). However, in the multivariate analysis using the Fine and Gray regression model with a competing risk of death, age $>60$ years and poor performance $(E C O G \geq 2)$ were found to be predictive variables for VTE (Table 3 ). 
A

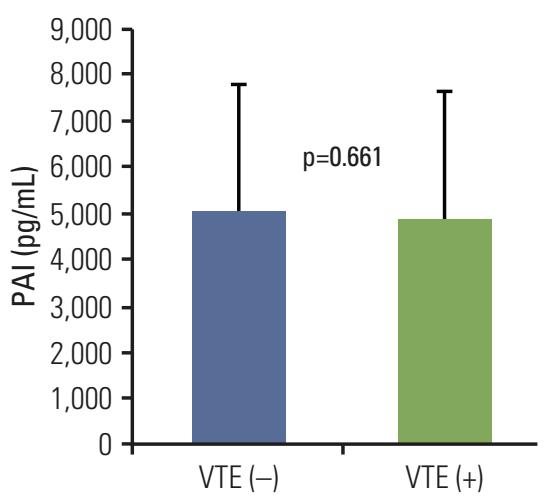

D

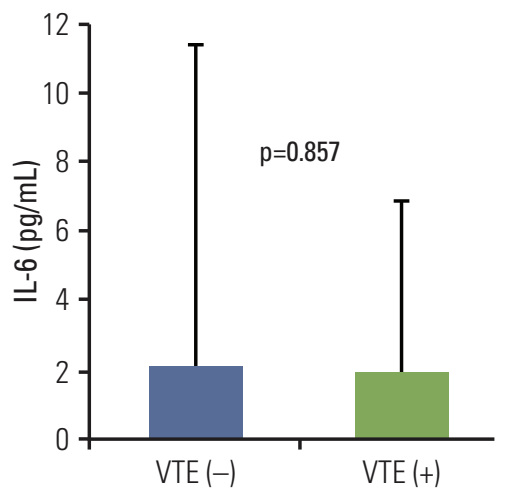

B

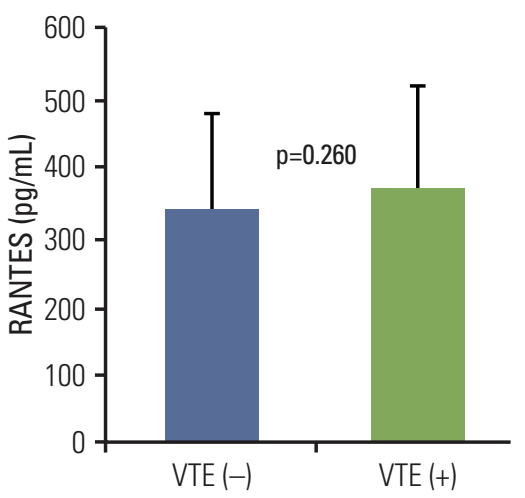

$\mathbf{E}$

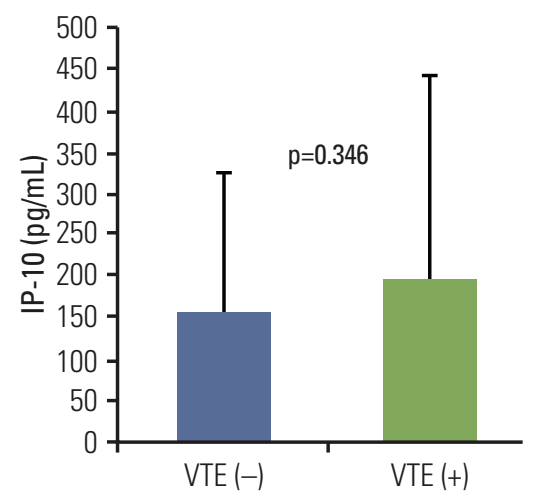

C

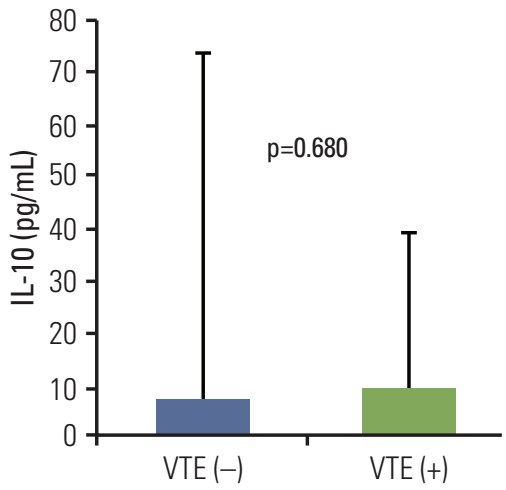

$\mathbf{F}$

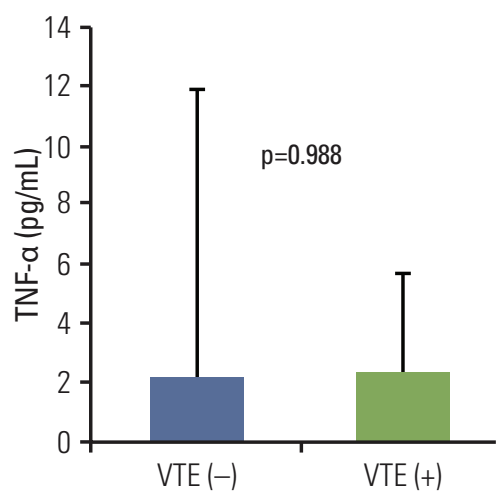

Fig. 3. Comparison of inflammatory biomarkers in patients with and without venous thromboembolism (VTE). The mean serum levels of inflammatory biomarkers plasminogen activator inhibitor-1 (PAI) (A), regulated on activation, normal T cell expressed and secreted (RANTES) (B), interleukin (IL) 10 (C), IL-6 (D), interferon gamma-induced protein 10 (IP-10) (E), and tumor necrosis factor $\alpha($ TNF- $\alpha)(F)$ were not significantly different between patients with and without VTE.

\section{Impact of VTE on overall survival}

Among 34 patients with VTE, eight died (8/34, 23.5\%). Two deaths were associated with VTE, and the other patients died because of disease progression. One relapsed patient died because of PE during salvage treatment. The other patient, who showed complete response after the third cycle of R-CHOP, developed acute dyspnea and hypotension from PE and died from uncontrolled bleeding caused by heparinization and with thrombocytopenia. However, the overall survival of patients with VTE was not significantly different from patients without VTE (Fig. 5).

\section{Discussion}

VTE was documented in 34 of the 322 patients $(10.6 \%)$ with newly diagnosed DLBCL; the 1-year actuarial incidence rate was $9.8 \%$. This incidence is slightly higher than that of our previous study, which found a crude incidence of $22 / 271$ $(8.1 \%)$ and a 1 -year actuarial incidence of $8.9 \%$ [8]. This increased incidence might be associated with the larger number of patients and the extended follow-up. Our incidence is similar to a Japanese study reporting VTE in 12.8\% of 211 patients with DLBCL [14]. Thus, VTE should be regarded as an important adverse event with significant incidence in Asian patients with DLBCL who receive systemic chemotherapy, as it is for other hematological and solid malignancies. In this study, we observed two VTErelated deaths. Although the overall survival of patients with 
Table 2. Competing risk factor analysis for VTE

\begin{tabular}{|c|c|c|c|}
\hline Variable & SHR & 95\% Confidence interval & p-value ${ }^{a)}$ \\
\hline Age $>60$ yr & 2.08 & $1.06-4.09$ & 0.03 \\
\hline Sex (female) & 0.89 & $0.44-1.77$ & 0.73 \\
\hline Smoking & 1.15 & $0.57-2.30$ & 0.69 \\
\hline Alcohol & 0.89 & $0.45-1.77$ & 0.75 \\
\hline $\mathrm{ECOG} \geq 2$ & 2.09 & $0.97-4.52$ & 0.06 \\
\hline Body mass index $\geq 25 \mathrm{~kg} / \mathrm{m}^{2}$ & 5.39 & $0.94-31.10$ & 0.06 \\
\hline B Symptom & 1.31 & $0.63-2.75$ & 0.47 \\
\hline Stage III/IV & 3.31 & $1.55-7.09$ & $<0.01$ \\
\hline No. of extranodal site $\geq 2$ & 2.88 & $1.47-5.66$ & $<0.01$ \\
\hline Increased LDH & 1.63 & $0.82-3.26$ & 0.17 \\
\hline IPI H/HI & 2.60 & $1.33-5.10$ & $<0.01$ \\
\hline Bone marrow involvement & 1.36 & $0.49-3.79$ & 0.55 \\
\hline Hemoglobin < $10 \mathrm{~g} / \mathrm{dL}$ & 1.58 & $0.70-3.55$ & 0.27 \\
\hline Platelet $\geq 350,000 \times 10^{9} / \mathrm{L}$ & 1.41 & $0.55-3.58$ & 0.48 \\
\hline $\mathrm{WBC}>11,000 / \mathrm{mm}^{3}$ & 2.78 & $1.12-6.86$ & 0.03 \\
\hline Radiotherapy & 1.16 & $0.44-3.11$ & 0.76 \\
\hline Surgery & 0.89 & $0.32-2.50$ & 0.83 \\
\hline Khorana score $\geq 3$ & 1.96 & $0.62-6.19$ & 0.25 \\
\hline $\mathrm{PAI} \geq$ median value & 1.58 & $0.97-2.58$ & 0.06 \\
\hline RANTES $\geq$ median value & 1.78 & $1.09-2.91$ & 0.02 \\
\hline IL- $10 \geq$ median value & 2.48 & $1.47-4.17$ & $<0.01$ \\
\hline IP-10 $\geq$ median value & 1.80 & $1.11-2.95$ & 0.02 \\
\hline IL-6 positive & 2.29 & $1.33-3.96$ & $<0.01$ \\
\hline TNF- $\alpha \geq 0.285 \mathrm{pg} / \mathrm{mL}$ & 0.77 & $0.46-1.29$ & 0.32 \\
\hline
\end{tabular}

VTE, venous thromboembolism; SHR, sub-distributional hazard ratio; ECOG, Eastern Cooperative Oncology Group; LDH, lactate dehydrogenase; IPI, International Prognostic Index; H, high; HI, high-intermediate; WBC, white blood cell; PAI, plasminogen activator inhibitor-1; RANTES, regulated on activation, normal T cell expressed and secreted, IL, interleukin; IP, interferon gamma-induced protein; TNF, tumor necrosis factor. ${ }^{\text {a) }}$ Gray's test.

VTE was not significantly different from that of patients without VTE (Fig. 3), the probability of a negative impact of VTE on treatment outcomes should be underscored.

Our study population had several characteristics that differ from previous studies. First, all patients were enrolled into the prospective cohort study, so their serum samples were archived at diagnosis. Second, patients had the same histological subtype and received one treatment regimen, $\mathrm{R}-\mathrm{CHOP}$, as the primary treatment. Third, all patients had a central venous catheter installed for chemotherapy. As a result, out of previously known risk factors for VTE, the presence of a central venous catheter and the use of chemotherapy were excluded from the analysis. Therefore, we could analyze the effect of host- and disease-related factors on the occurrence of VTE independent from central catheterization and the type of chemotherapy. In addition, we could analyze whether serum cytokines associated with inflammation and procoagulant proteins influence the occurrence of VTE.

In the univariate analysis with a competing risk of death, parameters from the Khorana score failed to show significant associations with VTE, except for the WBC count (Table 2). The Khorana risk score was proposed according to pretreatment hematology results, BMI and site of cancer as risk factors, and lymphoma is designated a high-risk cancer $[16,17]$. Although it was validated for the prediction of VTE in various patients with cancer from several studies [19], it might be limited when applied only to patients with lymphoma, because cytopenia rather than cytosis could be more common, especially in patients with bone marrow involvement or splenomegaly. Furthermore, as the BMI of Asian patients is usually less than $35 \mathrm{~kg} / \mathrm{m}^{2}$, this predictive model might have a limited value in this population. That is why it failed to predict the development of VTE in our study. On the other hand, the IPI risk model was more strongly associated with VTE than the Khorana score because parameters included in the IPI such as age, performance status, stage and extranodal involvement were significantly associated with VTE (Table 2). 
A

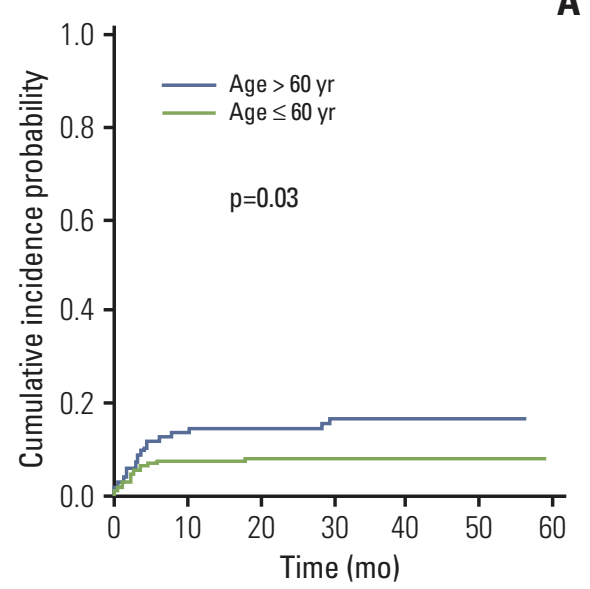

D

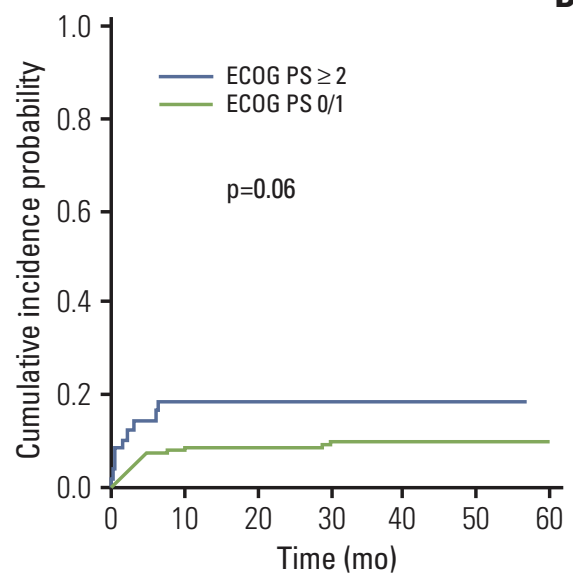

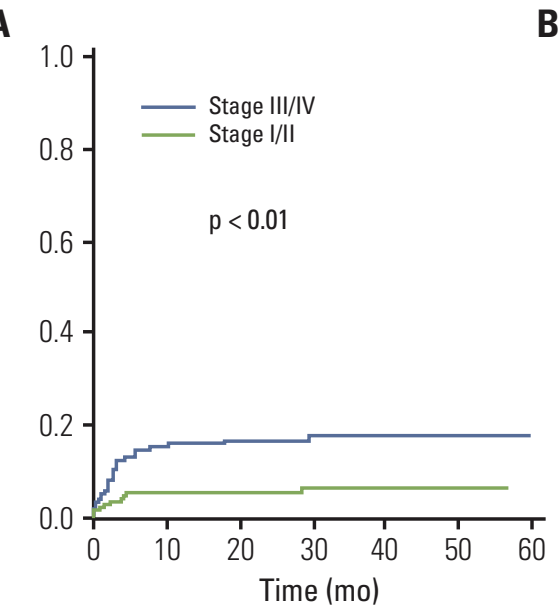

B

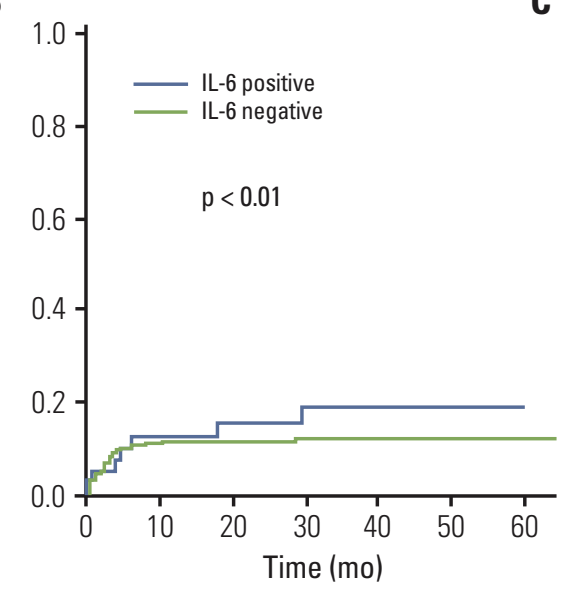

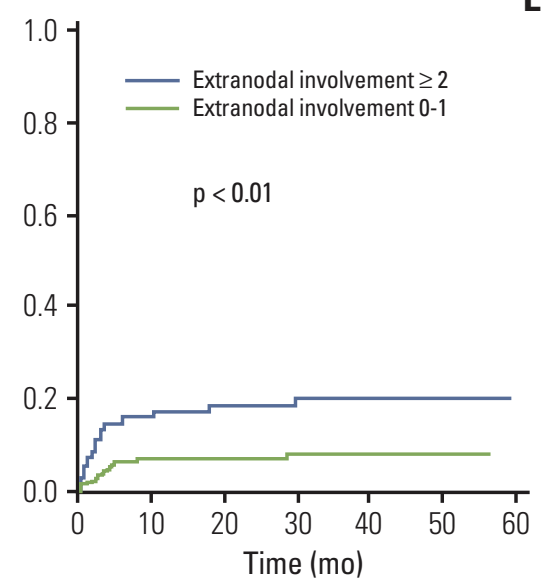

E

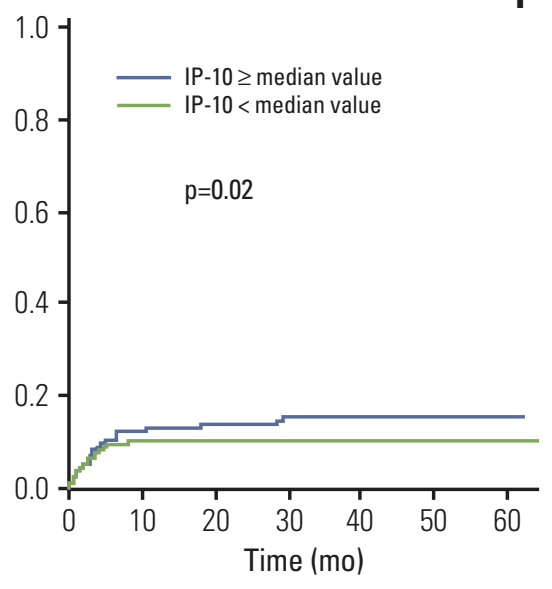

Fig. 4. Comparison of host factors, tumor burden and inflammatory cy tokines on the occurrence of venous thromboembolism (VTE). The occurrence of VTE was associated with old age (A), advanced stage (B), presence of interleukin (IL) 6 (C), poor PS (D), extranodal involvements (E), and elevated serum interferon gamma-induced protein 10 (IP-10) level (F). ECOG PS, Eastern Cooperative Oncology Group performance status.

Table 3. Competing risk factor multivariate analysis for VTE

\begin{tabular}{lcrr} 
Variable & SHR & 95\% Confidence interval & p-value \\
\hline Age $>60$ yr & 2.66 & $1.57-4.50$ & $<0.01$ \\
Stage III-IV & 1.18 & $0.57-2.43$ & 0.65 \\
\hline ECOG $\geq 2$ & 2.00 & $1.08-3.70$ & 0.03 \\
No. of extranodal site $\geq 2$ & 1.26 & $0.62-2.54$ & 0.52 \\
WBC $>11,000 / \mathrm{mm}^{3}$ & 1.33 & $0.56-3.15$ & 0.52 \\
PAI $\geq$ median value & 1.41 & $0.82-2.42$ & 0.21 \\
RANTES $\geq$ median value & 1.31 & $0.73-2.31$ & 0.36 \\
IL-10 $\geq$ median value & 1.66 & $0.84-3.26$ & 0.14 \\
IL-6 positive & 1.07 & $0.56-2.06$ & 0.84 \\
IP-10 $\geq$ median value & 0.82 & $0.43-1.55$ & 0.44 \\
\hline
\end{tabular}

VTE, venous thromboembolism; SHR, sub-distributional hazard ratio; ECOG, Eastern Cooperative Oncology Group; WBC, white blood cell; PAI, plasminogen activator inhibitor-1; RANTES, regulated on activation, normal T cell expressed and secreted, IL, interleukin; IP, interferon gamma-induced protein. 


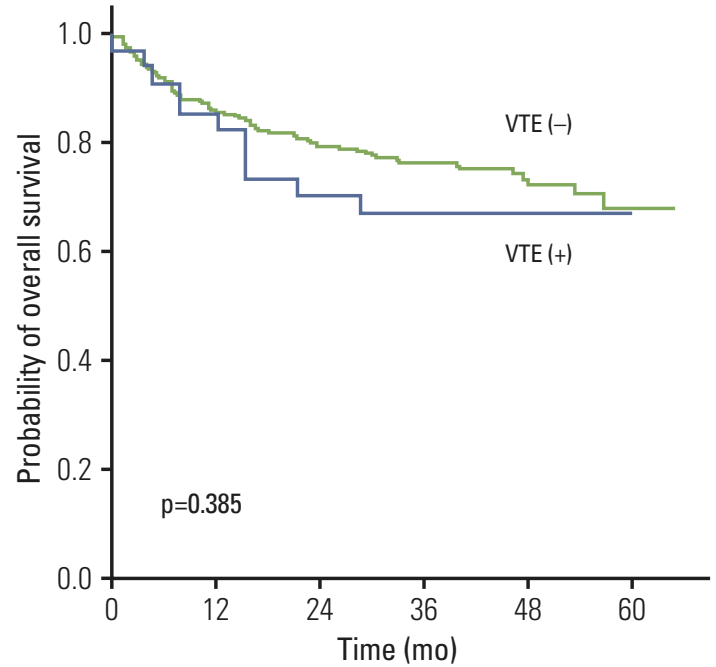

Fig. 5. Comparison of survival rates between patients with and without venous thromboembolism (VTE). The overall survival rate was not significantly different between patients with or without VTE.

Univariate analysis also indicated that the levels of several serum cytokines were associated with the occurrence of VTE (Table 2). Indeed, proinflammatory cytokines are commonly elevated in patients with lymphoma, and increased inflammatory activity worsens the prognosis for such patients. Likewise, proinflammatory cytokines might be associated with VTE because thrombosis can stimulate multiple inflammatory pathways and vice-versa. Possible mechanisms through which inflammation might promote a venous thrombosis are several. First, inflammatory cytokines can activate coagulation by the induction of tissue factor synthesis by blood monocytes, leading to activation of the extrinsic coagulation pathway. Second, thrombosis directly stimulates an inflammatory response in the vein wall, which involves neutrophil activation and expression of inflammatory cytokines and cellular adhesion molecules [20]. Although the mean values of the serum cytokines analyzed in this study did not differ significantly between patients with and without VTE, the dichotomization of patients by the cutoff value of those cytokines indicated that patients with high levels of RANTES, IL-10, IP-10, and IL-6 were more likely to develop VTE. This was consistent with studies showing the association of the levels of the proinflammatory cytokines IL- 6 and RANTES with VTE [21]. However, our results for TNF- $\alpha$ and IL-10 levels were contrary to other reports, which found proinflammatory cytokine TNF- $\alpha$ levels to be higher in patients with VTE, and immunoregulatory, anti-inflammatory cytokine IL-10 levels to be lower [22]. Most of those stud- ies were retrospective, and risk factor analysis was done without considering the competing risk of death from VTE. So far, no direct evidence supports the idea that these cytokines are predictive of the development of VTE or can determine the optimal cutoff value of each marker for estimating its occurrence [23]. Therefore, we could not demonstrate a clear association of these inflammatory cytokines with VTE in this study. The serum level of PAI-1 at diagnosis was not associated with VTE either in this study, although it had marginal significance $(\mathrm{p}=0.06$; hazard ratio, 1.58$)$ (Table 2). Given that the role of PAI is the inhibition of plasminogen activator, increased levels of PAI-1 inducing hypofibrinolysis might be associated with the occurrence of VTE. Indeed, the PAI-1 level is elevated in various disorders including cancer as well as in the aging process, and might contribute to the development of thrombosis [24]. Thus, the exact relationship between PAI-1 and VTE in patients with DLBCL requires further study.

Our multivariate analysis indicated that only age and performance status were independently associated with VTE. Our results are consistent with the general belief that frail elderly patients might be more vulnerable to adverse events, including VTE. However, this might also be interpreted to mean that patients with advanced disease and increased inflammation overlapped with patients older than 60 years with poor performance status, because patients with high tumor burden might have poorer performance status, and elderly patients are more likely to be diagnosed with an advanced disease.

\section{Conclusion}

In conclusion, our results suggest that age older than 60 years and poor performance status $(E C O G \geq 2)$ have a significant influence on the occurrence of VTE in patients with newly diagnosed DLBCL who received R-CHOP chemotherapy, more than other potential risk factors including disease and inflammation-related factors. Thus, careful monitoring and active intervention against VTE should be considered in elderly patients with DLBCL and poor performance status.

\section{Conflicts of Interest}

Conflict of interest relevant to this article was not reported. 


\section{Acknowledgments}

This study was supported by grants from the Samsung Biomedical Research Institute (GL1B30311), and the National
Research Foundation of Korea's (NRF) Basic Science Research Program (NRF-2014R1A2A1A11049853), which is funded by the Ministry of Education, Science and Technology.

\section{References}

1. Lekovic D, Miljic P, Mihaljevic B. Increased risk of venous thromboembolism in patients with primary mediastinal large B-cell lymphoma. Thromb Res. 2010;126:477-80.

2. Caruso V, Di Castelnuovo A, Meschengieser S, Lazzari MA, de Gaetano G, Storti S, et al. Thrombotic complications in adult patients with lymphoma: a meta-analysis of 29 independent cohorts including 18018 patients and 1149 events. Blood. 2010;115:5322-8.

3. Huh J. Epidemiologic overview of malignant lymphoma. Korean J Hematol. 2012;47:92-104.

4. Coiffier B. State-of-the-art therapeutics: diffuse large B-cell lymphoma. J Clin Oncol. 2005;23:6387-93.

5. Coiffier B, Lepage E, Briere J, Herbrecht R, Tilly H, Bouabdallah $\mathrm{R}$, et al. CHOP chemotherapy plus rituximab compared with $\mathrm{CHOP}$ alone in elderly patients with diffuse large-B-cell lymphoma. N Engl J Med. 2002;346:235-42.

6. Coiffier B, Thieblemont C, Van Den Neste E, Lepeu G, Plantier I, Castaigne $S$, et al. Long-term outcome of patients in the LNH-98.5 trial, the first randomized study comparing rituximab-CHOP to standard CHOP chemotherapy in DLBCL patients: a study by the Groupe d'Etudes des Lymphomes de l'Adulte. Blood. 2010;116:2040-5.

7. Yokoyama K, Murata M, Ikeda Y, Okamoto S. Incidence and risk factors for developing venous thromboembolism in Japanese with diffuse large b-cell lymphoma. Thromb Res. 2012;130:7-11.

8. Park LC, Woo SY, Kim S, Jeon H, Ko YH, Kim SJ, et al. Incidence, risk factors and clinical features of venous thromboembolism in newly diagnosed lymphoma patients: results from a prospective cohort study with Asian population. Thromb Res. 2012;130:e6-12.

9. Ferroni P, Riondino S, Formica V, Cereda V, Tosetto L, La Farina F, et al. Venous thromboembolism risk prediction in ambulatory cancer patients: clinical significance of neutrophil/lymphocyte ratio and platelet/lymphocyte ratio. Int J Cancer. 2015;136:1234-40.

10. Prandoni P, Falanga A, Piccioli A. Cancer and venous thromboembolism. Lancet Oncol. 2005;6:401-10.

11. Reitsma PH, Rosendaal FR. Activation of innate immunity in patients with venous thrombosis: the Leiden Thrombophilia Study. J Thromb Haemost. 2004;2:619-22.

12. Mause SF, von Hundelshausen P, Zernecke A, Koenen RR, Weber C. Platelet microparticles: a transcellular delivery system for RANTES promoting monocyte recruitment on endothelium. Arterioscler Thromb Vasc Biol. 2005;25:1512-8.

13. Lv W, Duan Q, Wang L, Gong Z, Yang F, Song Y. Gene expression levels of cytokines in peripheral blood mononuclear cells from patients with pulmonary embolism. Mol Med Rep. 2013;7:1245-50.

14. Komrokji RS, Uppal NP, Khorana AA, Lyman GH, Kaplan KL, Fisher RI, et al. Venous thromboembolism in patients with diffuse large B-cell lymphoma. Leuk Lymphoma. 2006;47: 1029-33.

15. Khorana AA, Francis CW, Culakova E, Kuderer NM, Lyman $\mathrm{GH}$. Frequency, risk factors, and trends for venous thromboembolism among hospitalized cancer patients. Cancer. 2007;110:2339-46.

16. Khorana AA, Kuderer NM, Culakova E, Lyman GH, Francis CW. Development and validation of a predictive model for chemotherapy-associated thrombosis. Blood. 2008;111:4902-7.

17. Khorana AA, Francis CW, Culakova E, Lyman GH. Risk factors for chemotherapy-associated venous thromboembolism in a prospective observational study. Cancer. 2005;104: 2822-9.

18. Schemper M, Smith TL. A note on quantifying follow-up in studies of failure time. Control Clin Trials. 1996;17:343-6.

19. Ay C, Dunkler D, Marosi C, Chiriac AL, Vormittag R, Simanek $\mathrm{R}$, et al. Prediction of venous thromboembolism in cancer patients. Blood. 2010;116:5377-82.

20. Fox EA, Kahn SR. The relationship between inflammation and venous thrombosis: a systematic review of clinical studies. Thromb Haemost. 2005;94:362-5.

21. van Aken BE, den Heijer M, Bos GM, van Deventer SJ, Reitsma $\mathrm{PH}$. Recurrent venous thrombosis and markers of inflammation. Thromb Haemost. 2000;83:536-9.

22. Poredos $P$, Jezovnik MK. In patients with idiopathic venous thrombosis, interleukin-10 is decreased and related to endothelial dysfunction. Heart Vessels. 2011;26:596-602.

23. Christiansen SC, Naess IA, Cannegieter SC, Hammerstrom J, Rosendaal FR, Reitsma PH. Inflammatory cytokines as risk factors for a first venous thrombosis: a prospective population-based study. PLoS Med. 2006;3:e334.

24. Incalcaterra E, Meli F, Muratori I, Corrado E, Amato C, Canino $\mathrm{B}$, et al. Residual vein thrombosis and onset of post-thrombotic syndrome: influence of the $4 \mathrm{G} / 5 \mathrm{G}$ polymorphism of plasminogen activator inhibitor-1 gene. Thromb Res. 2014;133:371-4. 